\title{
Geospace monitoring for space weather research and operation
}

\author{
Tsutomu Nagatsuma ${ }^{1, *}$ \\ ${ }^{1}$ Space Environment Laboratory, Applied Electromagnetic Research Institute, National Institute of \\ Information and Communications Technology, Japan, 184-8795, Tokyo, Koganei, Nukui-Kita, 4-2-1
}

\begin{abstract}
Geospace, a space surrounding the Earth, is one of the key area for space weather. Because geospace environment dynamically varies depending on the solar wind conditions. Many kinds of space assets are operating in geospace for practical purposes. Anomalies of space assets are sometimes happened because of space weather disturbances in geospace. Therefore, monitoring and forecasting of geospace environment is very important tasks for NICT's space weather research and development. To monitor and to improve forecasting model, fluxgate magnetometers and HF radars are operated by our laboratory, and its data are used for our research work, too. We also operate real-time data acquisition system for satellite data, such as DSCOVR, STEREO, and routinely received high energy particle data from Himawari-8. Based on these data, we are monitoring current condition of geomagnetic disturbances, and that of radiation belt. Using these data, we have developed empirical models for relativistic electron flux at GEO and inner magnetosphere. To provide userfriendly information, we are trying to develop individual spacecraft anomaly risk estimation tool based on combining models of space weather and those of spacecraft charging, Current status of geospace monitoring, forecasting, and research activities are introduced.
\end{abstract}

\section{Introduction}

It has been sixty years since the world first artificial satellite, Sputnik, was launched on Oct. 04, 1957. After that, space exploration is rapidly increasing, and more than several hundreds of artificial satellites are in orbit circulating around the Earth for practical purposes such as telecommunications, broadcasting, navigation, meteorology, etc. Thus, space assets are one of inevitable social infrastructure now. However, these space assets are vulnerable to space debris and space weather disturbances in geospace[1,2]. Based on the sixty years of space exploration, it was realized that the Sun is major driver of space disturbances around the Earth. The space environment around the Earth (geospace) dynamically varies depending on the solar wind conditions. To mitigate spacecraft anomalies, monitoring and forecasting of geospace is important. National Institute of Information and Communications Technology (NICT) is in charge of operational space

\footnotetext{
${ }^{*}$ Corresponding author: tnagatsu@nict.go.jp
} 
weather service in Japan as regional warning centre of International Space Environment Service (ISES) [3].

Because the solar-terrestrial relations are quite complicated, and the part of the physical processes in the relations are still unknown, global network of ground-based and spacebased observations needs to be preserved and improved. To contribute global space weather observation network, NICT have been operating regional ground-based observation network, and ground-based data reception facilities for space weather real time data stream from space-based observation. In this paper, NICT's activity about geospace monitoring for space weather research and operations are introduced.

\section{Ground-based observation}

Based on the international collaboration, NICT has constructed comprehensive groundbased networks for space weather monitoring along the Japanese meridian from the pole to the equator. These networks are used for operation and research of space weather forecasting [3]. For space weather monitoring, most of the data obtained from the networks are available in near-real time. These data are also useful for advanced research of space weather phenomena and for development of space weather simulations and models and its validation. Further, the data can be applied to future numerical space weather forecasting scheme based on data- assimilated simulation and modelling.

Current conditions of geomagnetic disturbances and ULF wave activities which contribute to acceleration of relativistic electron in geospace, are monitored by groundbased magnetometer networks and HF radar at King Salmon. Some of the magnetometers are operated by the international project Russian Auroral and Polar Ionospheric Disturbance Magnetometers (RapidMAG) [4]. Other magnetometers are operated under the research collaboration with IKIR in Russia. The HF radar at King Salmon is operated as a part of the Super Dual Auroral Radar Network (SuperDARN) [5].

EMIC waves play an important role for loss of relativistic electron in geospace. To monitor EMIC waves activity around sub-auroral region, we have installed induction magnetometers recently at sub-auroral region as part of project study of dynamical variation of Particles and Waves in the INner magnetosphere using Ground-based observations (PWING).

\section{Space-based observation}

Space-based observation is one of the key element for space weather monitoring and forecasting. However, there are limited number of operational satellite in orbit. Scientific satellites for basic research of solar-terrestrial physics contribute monitoring of space weather. Although NICT itself has no own spacecraft for space-based observations, two ground-based data reception facilities are operated for real-time space weather monitoring. One is for Advanced Composition Explorer (ACE) and Deep Space Climate Observatory (DSCOVR). The other is for Solar Terrestrial Relations Observatory (STEREO).

The L1 Lagrange point of the Sun-Earth system is one of the key locations for space weather monitoring. Because the L1 point is located about 1,500,000 km ahead of Earth toward the sun, the condition of the solar wind can be monitored with about one-hour leading time. NASA's Advanced Composition Explorer (ACE) was launched at the L1 point in 1997, and had the "real-time beacon mode" for broadcasting the solar wind and high-energy particle data in real time [6]. NICT has contributed to receiving the real-time beacon mode from the start of the ACE mission. In 2014, we renewed the spacecraft data reception system for ACE data acquisition because of the replacement of old facilities. This 
new data reception system can be applied to the coming real-time solar wind data from the Deep Space Climate Observatory (DSCOVR).

The Solar Terrestrial Relations Observatory (STEREO) is NASA's scientific mission for solar and interplanetary physics. A subset of the STEREO data is continuously downlinked in the real-time broadcast mode, called the Space Weather Beacon. The solar wind data (e.g., velocity, density, temperature, and vector magnetic fields) and low-resolution images of the solar EUV, coronagraph, and interplanetary space are contained in the Space Weather Beacon [7]. The data obtained from the STEREO Space Weather Beacon mode is used for estimating the recurrence structure of the solar wind, propagating direction of the interplanetary CMEs, and so forth.

Monitoring of high-energy particle environments in geospace is necessary for satellite operation, because high-energy particles are one of the major causes of spacecraft anomaly [8]. When some anomaly happens in a satellite, the operator's quick understanding of the current status of the space environment is needed for anomaly triage [9]. We have collected many types of space-based observations from GOES operational space weather data including high-energy protons and electron data in near real time. In addition, the Space Environment Data Acquisition Monitor (SEDA), which measures high-energy electrons and protons, is on board Himawari-8, a Japanese meteorological satellite in GEO. SEDA have been collected in near real time since Nov. 2014 [10]. Near-real-time SEDA data is provided by Japan Meteorological Agency (JMA) to NICT for the monitoring and forecasting of the space environment around a geostationary orbit. NICT plays roles in the processing and analyses of SEDA data. We are operating a quick-look web page of SEDA data and data server for real-time SEDA data distribution. A registered user will be able to download SEDA data in near real time.

The Energization and Radiation in Geospace (ERG) project is JAXA's science program for studying the acceleration and loss of radiation belt particles based on a small satellite, ground-based space weather observation networks, and computer simulations [11]. ERG was successfully launched on Dec. 20, 2016. ERG is providing real-time space weather data on high-energy particles and magnetic field measurements for the real-time monitoring of geospace. NICT is collecting these data for monitoring and forecasting of the space environment in geospace.

\section{Model and user-oriented information}

Spacecraft charging is one of the major causes of spacecraft anomalies. There are two types of spacecraft charging. One is surface charging, the other is deep charging. The surface charging is caused by electrons whose energy range is from several tens of $\mathrm{keV}$ to several hundreds of keV. Those energy range of electron flux is enhanced due to substorm activity. The deep charging is caused by electrons whose energy range is more than several hundreds of $\mathrm{keV}$. Those energy range of electron flux is enhanced due to variations of radiation belt. So we have been developed prediction model of geomagnetic disturbances including substorm activities, and radiation belt electron flux [12].

However, it is not easy to estimate risks of spacecraft anomalies only from space environment information. Because the threshold of charging condition depends not only on space environment, but also on materials and structure of the satellite itself. This means that the risk level of spacecraft anomaly is not the same for individual satellite. This means that nowcasting and forecasting information of space weather may not be sufficient for satellite operator. For this reason, we start developing a system to estimate the charging risk of individual satellites with different locations based on the combining forecast models of particle environment in geospace and multi-utility spacecraft charging analysis tool (MUSCAT)[13] and to promote the utilization of space weather forecast for satellite 
operators[14]. This activity is called as SECURES (Space Environment Customized Risk Estimation for Satellite) under Project for Space Terrestrial Prediction (PSTEP) (figure 1). We try to stimulate dialogues between satellite operator and space weather researcher and forecaster based on SECURES.

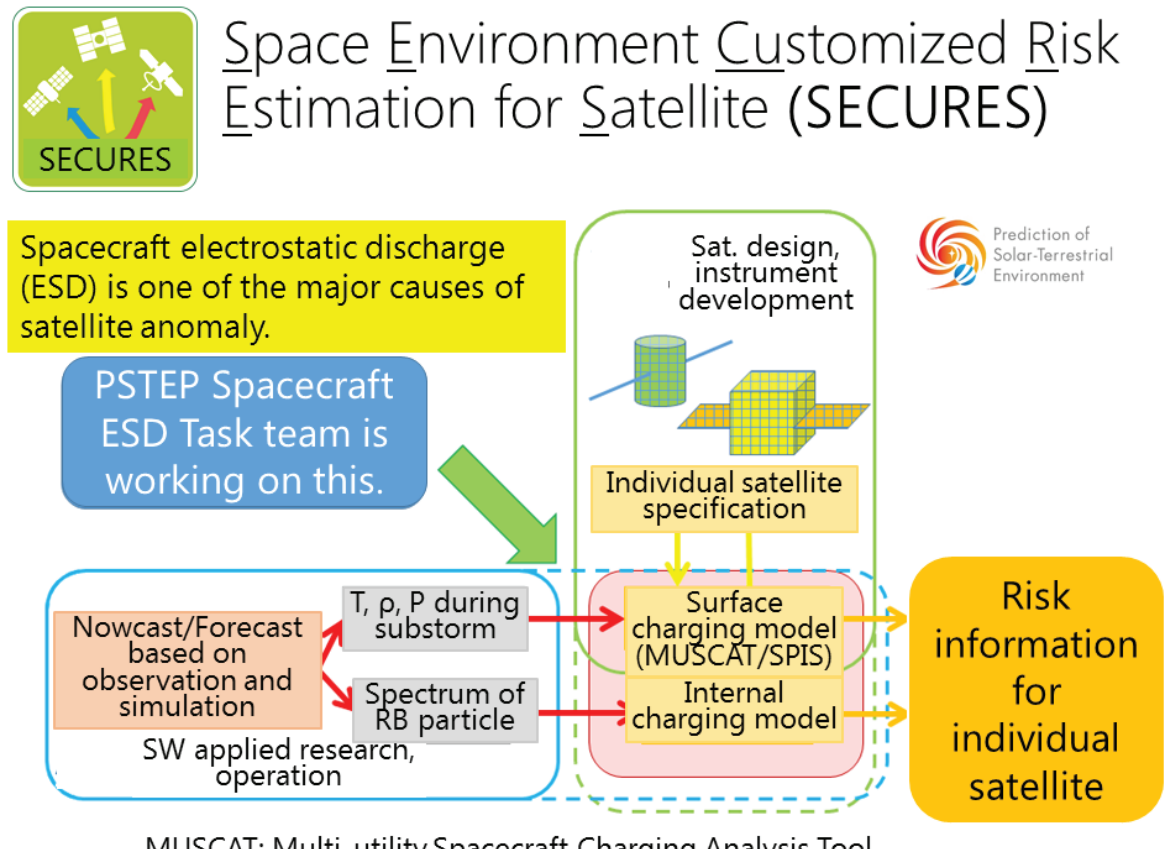

MUSCAT: Multi-utility Spacecraft Charging Analysis Tool

Fig. 1. Concept of SECURES (Space Environment Customized Risk Estimation for Satellite)

\section{Summary}

Geospace, a space surrounding the Earth, is one of the key area for space weather. Because geospace environment dynamically varies depending on the solar wind conditions. Many kinds of space assets are operating in geospace for practical purposes. Anomalies of space assets are sometimes happened because of space weather disturbances in geospace. Therefore, monitoring and forecasting of geospace environment is very important tasks for NICT's space weather research and development. To monitor and to improve forecasting model, fluxgate magnetometers and HF radars are operated by our laboratory, and its data are used for our research work, too. We also operate real-time data acquisition system for satellite data, such as DSCOVR, STEREO, and routinely received high energy particle data from Himawari-8. Based on these data, we are monitoring current condition of geomagnetic disturbances, and that of radiation belt. Using these data, we have developed empirical models for relativistic electron flux at GEO and inner magnetosphere. To provide userfriendly information, we are trying to develop SECURES, individual spacecraft anomaly risk estimation tool based on combining models of space weather and those of spacecraft charging. SECURES enables us to stimulate dialogues between satellite operator and space weather researcher and forecaster. 


\section{Acknowledgment}

Part of this work was supported by JSPS KAKENHI Grant Number JP15H05813 and JP16H06286.

\section{References}

1. Lanzerotti L.J., Breglia C., Maurer D.W., Johnson, G.K.III, Maclennan C.G., Adv. Space. Res., 22, 79-82, doi:10.1016/S0273-1177(97)01104-6 (1998)

2. Baker D.N., IEEE Trans. Plasma Sci., 28, 2007-2016 (2000)

3. Nagatsuma T., Space Weather, 11, doi :10.1002/swe.20050 (2013)

4. Takahashi K., Meng C.I., Kamei T., Kikuchi T., Kunitake M., Space Weather, 2, S11003, doi:10.1029/2004SW000116 (2004)

5. Chisham G., Lester M., Millan S.E., Freeman M.P., Bristow W.A., Grocott A., McWilliams K.A., Ruohoniemi J.M., Yeoman T.K., Dyson P.L., Greenwald R.A., Kikuchi T., Pinnock M., Rash J.P.S., Sato N., Sofko G.J., Villain J.-P., Walker A.D.M. Surv. Geophys. 28, 33-109 (2007)

6. Zwickl R.D. et al., Space Sci. Rev., 86, 633-648 (1998)

7. Biesecker D.A., Webb D.F., St. Cyr O.C., Space Sci. Rev., 136, 45-65 (2008)

8. Bodeau, J.M., paper AIAA 2010-1608 presented at 48th AIAA Aerospace Sciences Meeting Including the New Horizons Forum and Aerospace Exposition, Orland, Fla., doi :10.2514/6.2010-2010-1608 (2010)

9. O’Brien T.P., Mazur J.E., Fennell J.F., Space Weather, 11, 49, doi :10.1002/swe.20028 (2013)

10. Nagatsuma T., Sakaguchi K., Kubo Y., Belgraver P., Chastellain F., Muff R., Otomo T., Earth, Planets and Space, 69:75 doi:10.1186/s40623-017-0659-6 (2017)

11. Shiokawa K., et al., Adv. Space Res., 38, 8, 1861-1869 (2006)

12. Sakaguchi K., Miyoshi Y., Saito S., Nagatsuma T., Seki K., and Murata K.T., Space Weather, 11, 79-80, doi :10.1002/swe.20020 (2013)

13. Hatta S., Kim J., J. Plasma Fusion Res., 88, 92-95 (2012) (in Japanese)

14. Nagatsuma T., Aeronaut. Space Sci. Jpn., 65, 96-99 (2017) (in Japanese) 